\title{
Embedded Flexible Optical Shear Sensor
}

\author{
Jeroen Missinne* ${ }^{* \ddagger}$ Erwin Bosman ${ }^{\dagger \ddagger}$, Bram Van Hoe ${ }^{* \ddagger}$, Geert Van Steenberge ${ }^{* \ddagger}$, \\ Peter Van Daele ${ }^{\dagger \ddagger}$ and Jan Vanfleteren* ${ }^{* \ddagger}$ \\ ${ }^{*}$ Ghent University, ELIS Department, CMST, Belgium \\ Email: \{Jeroen.Missinne, Bram.VanHoe, Geert.VanSteenberge, Jan.Vanfleteren \}@elis.ugent.be \\ ${ }^{\dagger}$ Ghent University, INTEC Department, CMST, Belgium \\ Email: \{Erwin.Bosman, Peter.VanDaele\}@ugent.be \\ ${ }^{\ddagger}$ IMEC, CMST, Belgium
}

\begin{abstract}
Monitoring shear stresses is increasingly important in the medical sector, where the sensors need to be unobtrusive, compact and flexible. A very thin and flexible sensor foil is presented based on the shear stress dependent coupling change of optical power between a laser and photodiode chip that were separated by a deformable sensing layer. These opto-electronic components were embedded in a very thin foil of only $40 \mu \mathrm{m}$ thick. The sensitivity and measurement range can be modified by selecting the material properties of the sensing layer. The sensor response showed to be reproducible and the influence of normal pressure on the sensor was very limited.
\end{abstract}

\section{INTRODUCTION}

Most of the existing shear sensors were originally intended to be used for monitoring fluid dynamics [1]. However, there is an increasing need for monitoring shear stresses in the medical field, for example for measuring the skin friction between prosthesis and stump [2]. Excessive stresses on the stump may damage the skin tissue causing discomfort for the amputee. Similarly, the shear stresses play an important role in the friction between foot and shoe [3] and also in the field of robotics there is and interest in tactile shear sensors for artificial skin. For these applications, the sensors are preferably as unobtrusive as possible and need to be compact and flexible to be used on moving body parts and wrapped around curved surfaces, such as fingers or prostheses.

A number of reported shear sensors are based on MEMS [4] which can have a high density and sensitivity but only have limited flexibility or are rather thick. They are also based on electrical measurements and thus sensitive to electromagnetic interference. Optical sensors are not susceptible to this source of noise and can have a number of advantages such as high sensitivity and large dynamic range. Furthermore they can be embedded which makes these sensors potentially very compact, robust and flexible.

The technology for embedding optical components into $40 \mu \mathrm{m}$ thick flexible foils was reported previously [5] and is now used to construct an optical shear sensor.

\section{SEnsor PRINCIPLE}

The shear sensor principle is based on the changing amount of optical power emitted by a Vertical-Cavity Surface-Emitting Laser (VCSEL) and detected by a photodiode. Both components are aligned with their active areas facing each other.

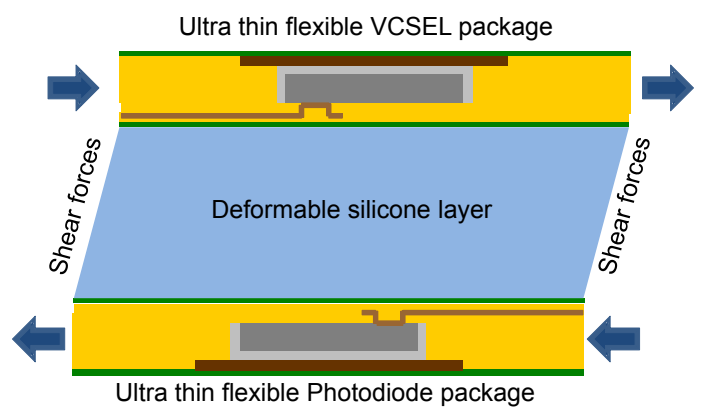

Fig. 1. Schematic view of the sensor principle

When shear stresses are applied onto the structure, a misalignment of the VCSEL and photodiode occurs and a varying amount of power is detected by the photodiode. A VCSEL and a photodiode were assembled in an ultra thin package [5] and separated by a deformable polymer sensing layer such as Polydimethylsiloxane (PDMS). The thickness and the mechanical properties of the separation layer determine the sensor characteristics, since the displacement of the VCSEL and photodiode under shear stress depends on this material behavior. In Fig. 1, this principle is depicted schematically.

The opto-electronic components used in this paper were bare die $850 \mathrm{~nm}$ multimode VCSEL chips from ULM Photonics [6] and photodiodes with a $100 \mu \mathrm{m}$ active area from Enablence [7].

\section{Simulations}

There are several design parameters to be chosen. Most important is the distance between VCSEL en photodiode, along with the divergence angle of the laser beam. To construct a simple analytical model, the VCSEL beam was considered Gaussian with a certain half power divergence angle that was defined as the single sided angle wherein $50 \%$ of the optical power is confined. However this assumption is not completely valid since multimode VCSELs were used for the fabrication of the sensor, it yields an adequate approximation. The movement of the photodiode over the VCSEL beam can be mathematically considered as calculating the convolution $\Phi_{d}(x, y) * P D(x, y)$ of a uniform circular detector area $P D(x, y)$ with the Gaussian VCSEL beam 


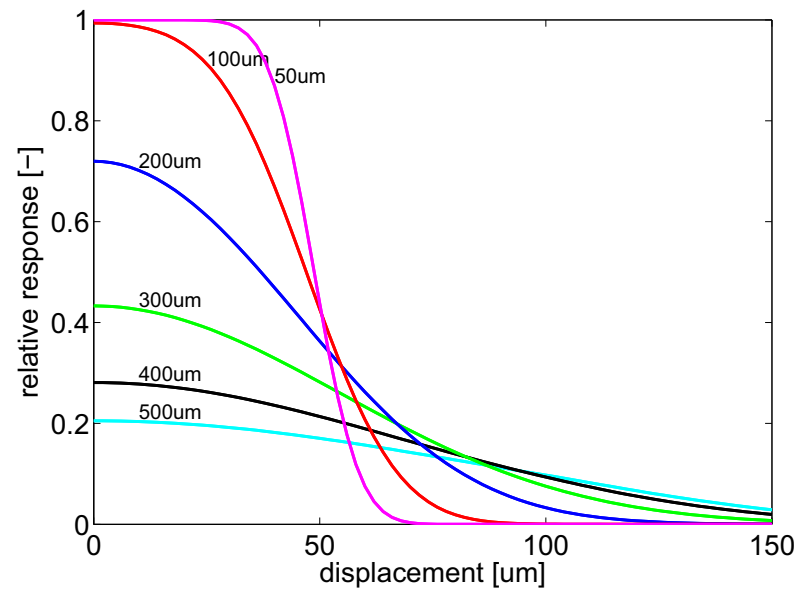

Fig. 2. Simulation of the photodiode current versus the displacement of the VCSEL for different VCSEL-to-photodiode distances (constant driving current $=5 \mathrm{~mA}$ )

profile $\Phi_{d}(x, y)$, considered at the specified distance $d$ from the detector. This yields the 2-dimensional relative sensor response.

relative response $=\iint \Phi_{d}\left(x^{\prime}, y^{\prime}\right) P D\left(x-x^{\prime}, y-y^{\prime}\right) d x^{\prime} d y^{\prime}$

All functions were centered around the origin. The resulting convolution yields the response for every possible displacement $(x, y)$ from the origin. For easy comparison, the 1-dimensional sensor response was considered and obtained by evaluating this expression for $y=0$.

The total optical power and beam divergence angles of the VCSELs at different driving currents were measured to make a realistic scaling of the beam profiles used in the simulations. The optical power in the VCSEL beam at $5 \mathrm{~mA}$ driving current was set to 1 as a reference and for the other currents, the values were scaled accordingly, based on the measurements. Also the detector function $P D(x, y)$ was normalized.

For easy interpretation of the simulation results, the displacement $x$ of the VCSEL with respect to the photodiode was chosen as input parameter instead of the shear stress applied onto the sensor. The displacement and shear stress are connected through the material properties of the sensing layer.

\section{A. Influence of VCSEL-to-detector distance}

In a first simulation, the influence of the VCSEL-to-detector distance was determined for a driving current of $5 \mathrm{~mA}$ (Fig. 2). Since the beam diverges, the response will be spread out more when the VCSEL and detector are separated further away. When the distance between source and detector is too small, the laser beam is relatively confined and almost all optical power is captured by the detector when aligned and none is captured when misaligned. In the transition region there is a steep response. The other extreme, where VCSEL and detector are far away from each other, seems favorable at first

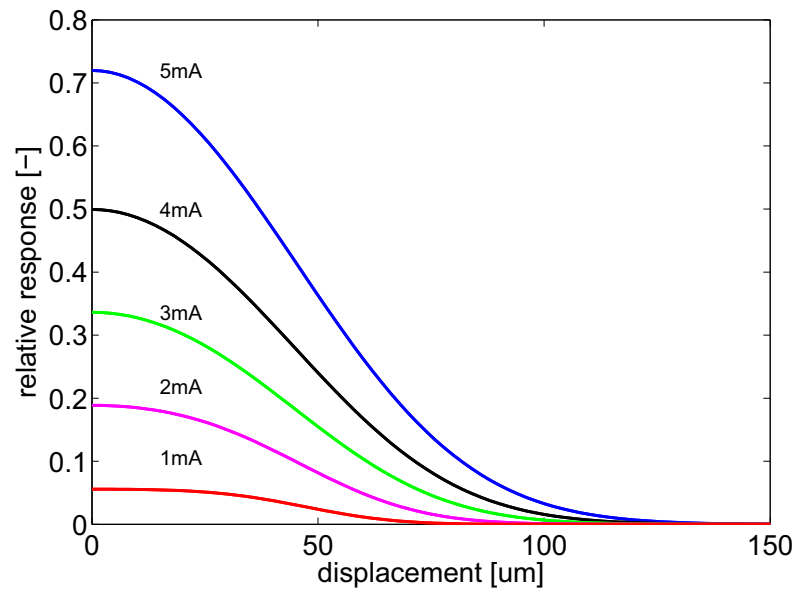

Fig. 3. Simulation of the photodiode current versus the displacement of the VCSEL for different driving current (constant VCSEL-to-detector distance of $200 \mu \mathrm{m})$

glance. However, the sensitivity and consequently the accuracy is low, particularly in the region close to zero displacement. Furthermore, this simple model does not completely reflect the real behavior. A typical multimode beam will not be Gaussian, but more power will be concentrated in the outer part of the beam. This effect will be averaged out when calculating the convolution, provided that the beam is not too wide or accordingly the distance to the detector not too large. Therefore a separation of $200 \mu \mathrm{m}$ seems a good compromise with a sensing range up to $100 \mu \mathrm{m}$ displacement.

\section{B. Influence of the VCSEL driving current}

The second simulation shows the influence of the driving current for a fixed VCSEL-to-detector distance of $200 \mu \mathrm{m}$. The variations in the sensor responses are due to the different beam divergence angles and optical power of the VCSELs depending on the current. For a driving current around the threshold, the half power divergence angle was measured to be $3^{\circ}$, while at $5 \mathrm{~mA}$ this was increased to $6^{\circ}$. Since the detector behaves as an averaging function for the beam profile of the VCSEL, the response has less variations for small driving currents since the VCSEL beam is almost completely incident to the detector for the largest part of the displacement. When the divergence angle increases, the detector is not large enough to capture all the light, yielding a more favorable sensor response.

\section{Influence of the sensing layer material properties}

For the conversion of displacement measurements $x$ into shear stress measurements, the material properties of the deformable layer have to be taken into account. A softer material yields a higher sensitivity while a stiffer material yields a higher shear stress sensing range. To be consistent with the simulations above, a PDMS thickness $d_{P D M S}$ of $180 \mu \mathrm{m}$ was chosen, since there was an additional packaging cover layer of $10 \mu \mathrm{m}$ on top of the VCSEL and photodiode active areas. The total VCSEL-to-detector distance is then $200 \mu \mathrm{m}$. 


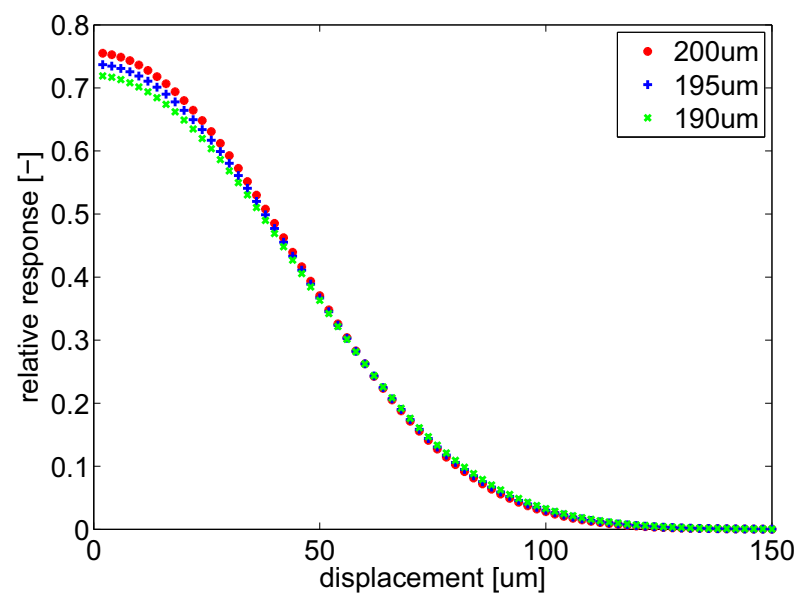

Fig. 4. Simulation of the sensor response for different values of VCSEL-todetector distance induced by a normal load on the sensor

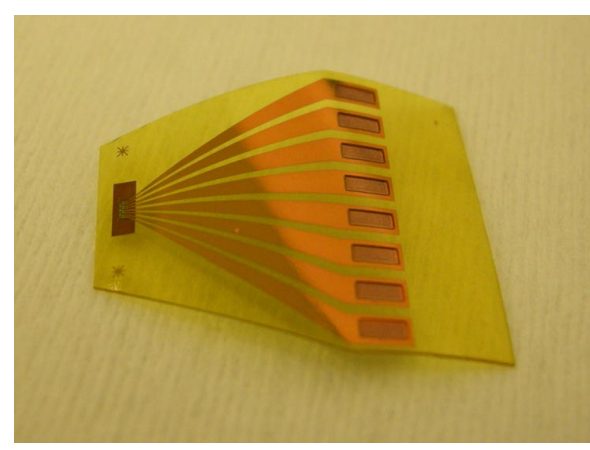

Fig. 5. A flexible ultra thin VCSEL package

The typical shear modulus $G$ of PDMS is about $250 \mathrm{kPa}$ [8], meaning that the shear stress $\tau_{x}$ and strain $\gamma_{x}$ are related as follows:

$\tau_{x}=G \gamma_{x}=G \cdot x / d_{P D M S}=250 \mathrm{kPa} \cdot x / 180 \mu \mathrm{m}$

which yields a maximum measurable shear stress of:

$\tau_{x}=250 \mathrm{kPa} \cdot 100 \mu \mathrm{m} / 180 \mu \mathrm{m} \approx 139 \mathrm{kPa}$

\section{Influence of the normal pressure}

Since the Poisson's ratio of PDMS is close to 0.5 , the material is considered almost incompressible and the influence of the normal pressure will be limited. Under typical normal loading conditions up to a few bar, the PDMS will be compressed by a few micrometers which also changes the distance between the VCSEL and the photodiode with the same amount. The influence of this PDMS compression on the sensor response is limited, as demonstrated in Fig. 4.

\section{FABRicATION METHODS}

The fabrication of the sensor is based on the packaging of opto-electronic components such as VCSELs and photodiodes

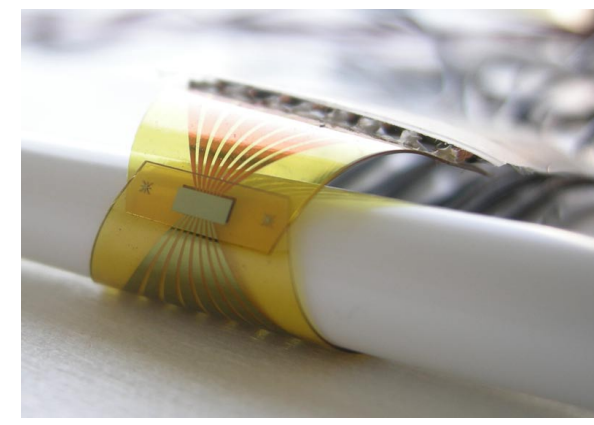

Fig. 6. The flexible shear sensor (bent with a radius of $4 \mathrm{~mm}$ ) based on ultra thin VCSEL and photodiode packages with a deformable PDMS sensing layer

into very thin and mechanically flexible packages and was previously reported [5]: bare dies were thinned down to $20 \mu \mathrm{m}$ and subsequently embedded in optically transparent layers, yielding a flexible package of only $40 \mu \mathrm{m}$ thickness (Fig. 5). Such a package, while still on a temporary glass carrier, was used as a starting point for the fabrication.

First, a $90 \mu \mathrm{m}$ layer of Dow Corning Sylgard $\mathrm{R} 184$ PDMS was spin-coated onto both the VCSEL and photodiode package, using a primer to obtain decent adhesion. The PDMS was subsequently cured on a hotplate at $60^{\circ} \mathrm{C}$ for 1 hour. A plasma bonding technique was used to join both PDMS layers. The substrates were exposed to an air plasma $(0.8 \mathrm{mbar}, 24 \mathrm{~s}, 190 \mathrm{~W}$ $40 \mathrm{kHz}$ generator) and afterwards the VCSEL and photodiode packages were aligned such that the active areas were facing each other. Finally, the PDMS layers were brought into contact to obtain bonding between both parts. The result is a highly flexible and compact sensor foil (Fig. 6).

Furthermore, this sensor design can potentially be used to fabricate high density sensing foils since the bare die embedded components are very small and packaged in a very thin foil.

\section{RESULTS AND DISCUSSION}

First tests were done to characterize the response of the VCSEL displacement versus photodiode in order to compare with the simulations. On the available shear sensor sample, the embedded photodiode had an active area of $100 \mu \mathrm{m}$ while the VCSEL was from the multimode type emitting at $850 \mathrm{~nm}$. Furthermore, the total thickness of the PDMS layer between both components was about $180 \mu \mathrm{m}$. The sensor was mounted on a translation stage and stretched to simulate shear stresses. For different driving currents of the VCSEL, the photodiode current with respect to the displacement was recorded. Therefore, the photodiode was inversely polarized at $-2 \mathrm{~V}$ and the photocurrent was measured without amplification.

A range of VCSEL driving currents, starting from the threshold of $1.5 \mathrm{~mA}$ up to $5 \mathrm{~mA}$ were tested. The results are plotted in Fig. 7. It can be seen from the plot that there seems to be a small difference in response between increasing and decreasing displacement, although it is believed that this behavior can be attributed to non-perfect clamping during testing rather than hysteresis. 


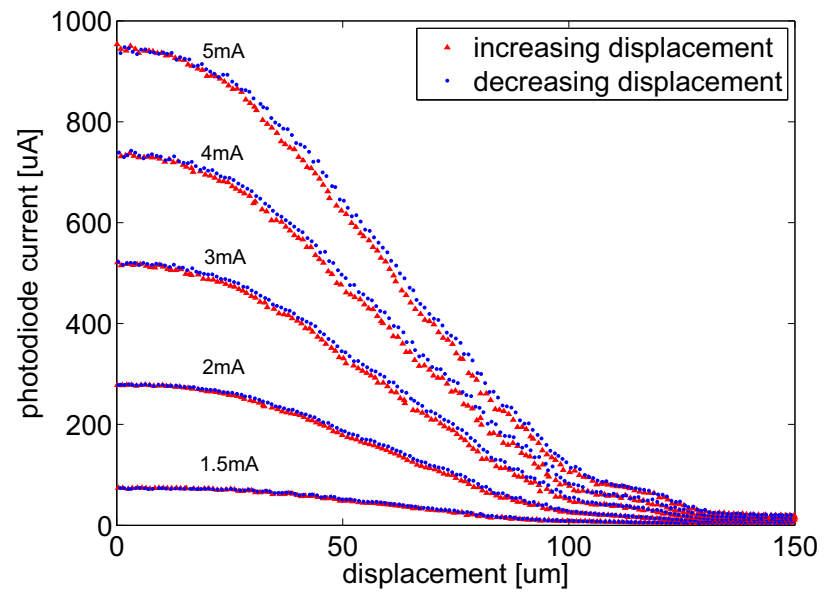

Fig. 7. Photodiode current versus the displacement of the VCSEL for different driving currents

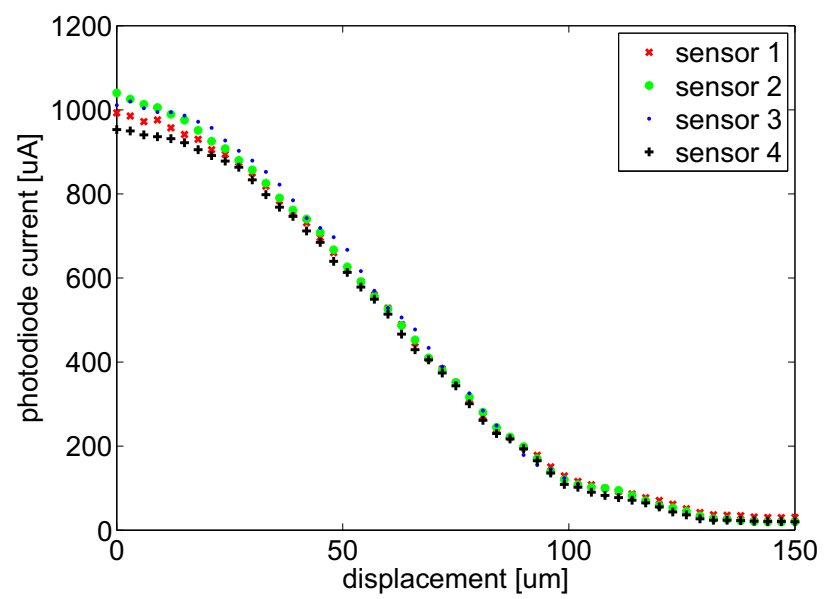

Fig. 8. Photodiode current versus the displacement of the VCSEL for different sensor points (driving current $5 \mathrm{~mA}$ )

The sensor response is clearly dependent on the driving current through the VCSEL. At lower driving currents, the laser beam is less powerful and the opening angle lower, yielding less sensitivity and range. A driving current of $5 \mathrm{~mA}$, however, gives a better range and good sensitivity. This behavior is in accordance with the simulation result from Fig. 3. Furthermore, the shape of the sensor response is similar to those from the simulations. Some deviations might be due to fabrication tolerances and the non-perfect Gaussian behavior of the used multimode VCSELS.

For a driving current of $5 \mathrm{~mA}$, the variation in response of different sensor points was measured (Fig. 8). The horizontal shift resulting from differing setup mounting offsets was eliminated. It can be seen that there is little variation between different sensors, suggesting that the beam profile is not significantly different between different sources. Furthermore, there is a quite linear sensor response from $25 \mu \mathrm{m}$ up to $100 \mu \mathrm{m}$ relative displacement. In this region, the sensitivity of the sensor without amplification of the photodiode current is $-11 \mu \mathrm{A} / \mu \mathrm{m}$. When taking into account the material properties, the sensitivity for measuring shear stress is:

$\frac{-11 \mu \mathrm{A}}{\mu \mathrm{m}} \cdot \frac{x}{\tau_{x}}=\frac{-11 \mu \mathrm{A}}{\mu \mathrm{m}} \cdot \frac{d}{G}=\frac{-11 \mu \mathrm{A}}{\mu \mathrm{m}} \cdot \frac{180 \mu \mathrm{m}}{250 \mathrm{kPa}}=-7.9 \frac{\mu \mathrm{A}}{\mathrm{kPa}}$.

\section{CONCLUSION}

In this paper, a new concept for measuring shear stresses has been demonstrated. The proposed optical sensor is based on the shear stress induced displacement of a VCSEL with respect to a photodiode separated by a thin PDMS layer. Both VCSEL and photodiode were used as bare dies and packaged in ultra thin flexible packages of only $40 \mu \mathrm{m}$ thick. This makes the sensor potentially very useful for medical applications where conformable and unobtrusive sensors are desirable.

Simulations were performed to optimize the sensor response and measurements confirmed this predicted behavior. Furthermore, it was found that the influence of the normal pressure on the shear stress response remains relatively low.

With the current design, shear stresses up to $139 \mathrm{kPa}$ can be measured with a sensitivity of $-7.9 \frac{\mu \mathrm{A}}{\mathrm{kPa}}$ in the linear part of the range. However, by using different sensing materials, the sensitivity and range can be tuned depending on the application.

\section{ACKNOWLEDGMENT}

This work is partially conducted in the framework of the projects FAOS (funded by the Institute for the Promotion of Innovation by Science and Technology (IWT), Flanders, Belgium) and Phosfos (funded within the EU-FP7 program). The work of J. Missinne is supported by the Research Foundation - Flanders (FWO-Vlaanderen) under a Ph. D. fellowship.

\section{REFERENCES}

[1] J. W. Naughton and M. Sheplak, "Modern developments in shear-stress measurement," Progress in Aerospace Sciences, vol. 38, no. 6-7, pp. 515 $-570,2002$.

[2] M. Zhang, A. R. Turner-Smith, A. Tanner, and V. C. Roberts, "Clinical investigation of the pressure and shear stress on the trans-tibial stump with a prosthesis," Medical Engineering \& Physics, vol. 20, no. 3, pp. 188 - 198, 1998.

[3] R. Hosein and M. Lord, "A study of in-shoe plantar shear in normals," Clinical Biomechanics, vol. 15, no. 1, pp. 46 - 53, 2000.

[4] K. Kim, K. R. Lee, D. S. Lee, N.-K. Cho, W. H. Kim, K.-B. Park, H.-D. Park, Y. K. Kim, Y.-K. Park, and J.-H. Kim, "A silicon-based flexible tactile sensor for ubiquitous robot companion applications," in Journal of Physics: Conference Series, vol. 34, no. 1. UK: IOP Publishing, 2006, Conference Paper, pp. 399-403, International MEMS Conference 2006, 9-12 May 2006, , Singapore.

[5] E. Bosman, G. Van Steenberge, B. Van Hoe, J. Missinne, J. Vanfleteren, and P. Van Daele, "Highly reliable flexible active optical links," IEEE Photon. Technol. Lett., vol. 22, no. 5, pp. 287-9, 1 March 2010.

[6] U.-L.-M. photonics. (2010) 850nm multimode bare die vcsel array chip. [Online]. Available: http://www.ulm-photonics.com/docs/products_new/ datasheets/VCSEL-ULM850-05-arraychip.pdf

[7] Enablence. $850 \mathrm{~nm}$ pin photodiode bare die chip array [Online]. Available: http://www.enablence.com/components-subsystems/ product-line/photodiodes

[8] J. Lotters, W. Olthuis, P. Veltink, and P. Bergveld, "The mechanical properties of the rubber elastic polymer polydimethylsiloxane for sensor applications," Journal of Micromechanics and Microengineering, vol. 7, no. 3, pp. 145-147, Sept 1997, 7th Workshop on Micromachining, Micromechanics and Microsystems in Europe (MME 96), Barcelona, Spain, Oct 21-22, 1996. 